\title{
Ações de controle populacional de cães não domiciliados realizadas pelo poder público em municípios do Rio de Janeiro, Brasil (2012-2013)*
}

\section{Populational control actions for stray dogs carried out by the public authorities in municipalities of Rio de Janeiro, Brazil (2012-2013)}

\author{
Flavio Fernando Batista Moutinho, ${ }^{* *}$ Elmiro Rosendo do Nascimento, ${ }^{* *}$ Rita Leal Paixão***
}

\begin{abstract}
Resumo
A grande densidade populacional de cães não domiciliados é uma realidade nos municípios brasileiros, trazendo problemas à ordem urbana e à saúde coletiva. Este trabalho descreve as ações desenvolvidas pelos órgãos de saúde dos municípios do Rio de Janeiro, no período 2012-2013, visando ao controle populacional de cães não domiciliados. Após a seleção de uma amostra estatisticamente significativa, composta por 47 municípios, houve a aplicação de questionário aos responsáveis pelos serviços de controle de zoonoses desses municípios. Somente $46,8 \%(n=22)$ realizavam alguma ação relacionada ao controle populacional. As principais ações desenvolvidas eram as atividades educativas sobre guarda responsável, a esterilização gratuita e os projetos de adoção, mesmo assim presentes em menos de $30 \%$ dos municípios. Já o recolhimento sistemático com posterior eutanásia não é mais uma prática frequente no Estado do Rio de Janeiro, não tendo sido relatado por nenhum município avaliado. De modo geral as poucas ações visando ao controle populacional de cães não domiciliados desenvolvidas pelos municípios avaliados, em todas as Regiões de Saúde, eram fragmentadas e não seguiam um protocolo de atuação com ações integradas.
\end{abstract}

Palavras-chave: vigilância em saúde; população canina; eutanásia; educação em saúde.

\begin{abstract}
The great populational density of stray dogs is a reality in the Brazilian municipalities, bringing problems to the urban order and the collective health. This paper describes the actions developed the health agencies of the municipalities of Rio de Janeiro, Brazil, in the period 2012-2013, seeking the populational control of stray dogs. After the selection of a statistically significant sample, comprised of 47 municipalities, a questionnaire was applied to those responsible for the zoonoses control services of these municipalities. Only $46.8 \%(n=22)$ of the municipalities performed some action related to the populational control. The main activities developed were the educational activities on responsible ownership, free sterilization and the adoption projects, even so, present in less than $30 \%$ of the municipalities. Now the systematic collection with later euthanasia is no longer a frequent practice in the State of Rio de Janeiro, not having been reported by any municipality evaluated. In general, the few actions seeking the populational control of stray dogs developed by the municipality evaluated, in all the Health Regions, were fragmented and did not follow a performance protocol with integrated actions.
\end{abstract}

Keywords: health surveillance; canine population; euthanasia; health education.

\section{Introdução}

Quase todos os municípios do país enfrentam problemas envolvendo animais, tais como crias indesejadas, abandono, superpopulação, criação e comercialização irregular ou ausência de regras para tal, denúncias de maus-tratos, mordeduras e desconhecimento e não utilização de preceitos de bem-estar. O problema das populações de cães não domiciliados é muito antigo e a criação de leis relacionadas ao controle de populações animais teve início na época de Pasteur, sob a influência das conclusões de seus estudos envolvendo a descoberta dos agentes etiológicos causadores de uma série de doenças, muitos dos quais tendo os animais domésticos como reservatórios (VIEIRA et al, 2006). O Brasil também foi influenciado por esses estudos, o que levou à criação da primeira lei sobre o assunto em 1895, promulgada pela Câmara Municipal de São Paulo, e que previa a proibição dos cães andarem soltos nas ruas, a obrigatoriedade de mordaça e de coleira com identificação (SOUZA, 2011).

Ainda que o problema da superpopulação de cães não domiciliados seja complexo e multifatorial e exija ações de enfrentamento multidisciplinares e multisetoriais é importante reconhecer que o controle de populações caninas requer

\footnotetext{
*Recebido em 25 de maio de 2015 e aceito em 11 de setembro de 2017.

${ }^{* *}$ Faculdade de Veterinária da Universidade Federal Fluminense, Niterói, RJ - Brasil.

***Instituto Biomédico da Universidade Federal Fluminense, Niterói, RJ - Brasil.

Autor para correspondência: flaviomoutinho@id.uff.br
} 
o conhecimento sobre a densidade ecológica, que vai ser influenciada pela entrada (nascimentos e dispersão) e saída (morte e dispersão) de indivíduos (FORATTINI, 2004). Além disso, é importante destacar que o processo reprodutivo dos animais de vida livre, como os cães não domiciliados, ocorre em um contexto influenciado pelas características sociais e territoriais da espécie (HAFEZ, 1995). Nesse contexto, uma única cadela pode gerar novos filhotes a cada 6 meses, com uma gestação média de 58 a 64 dias (GOMES, 2010).

Atualmente, o controle da população de cães não domiciliados é considerado necessário por questões de saúde coletiva, de bem-estar animal e de ordem urbana. A grande quantidade de cães soltos nas ruas pode provocar acidentes de trânsito, destruição dos patrimônios público e privado, maus-tratos contra estes animais, doenças transmissíveis (com ênfase para a raiva), e agressões a pessoas (REICHMANN et al, 2000).

Importante salientar que a Organização Mundial de Saúde (OMS), através do seu $6^{\circ}$ Informe Técnico, de 1973, recomendava o extermínio dos cães não domiciliados como forma de controlar as populações destes animais e evitar, como consequência, a ocorrência de antropozoonoses como a raiva. Durante muitos anos, a captura e posterior eutanásia de cães não domiciliados foram utilizadas de maneira indiscriminada e sistemática como forma de controle da população de cães em todo o mundo, inclusive no Brasil (SANTANA et al, 2008). A partir da década de 1990, após a realização de aprofundada pesquisa, a OMS passou a considerar que os programas de eliminação de cães por intermédio da captura e posterior eutanásia eram caros e ineficazes, não havendo comprovação de que as ações tenham gerado impacto significativo na redução da densidade de cães e na redução da propagação da raiva. Isso é justificado porque a rápida taxa de renovação da população canina e a taxa de sobrevivência sobrepõem com facilidade a taxa de eliminação destes animais. Além disso, há uma discussão bioética envolvida na eliminação de animais sadios (ORGANIZAÇÃO MUNDIAL DA SAÚDE, 1999).

No Estado do Rio de Janeiro é proibida a eutanásia como estratégia de controle populacional de cães e gatos e é previsto em lei que programas de esterilização permanentes devem ser realizados pelo Poder Público visando o controle reprodutivo destes animais (RIO DE JANEIRO, 2006).

Importante destacar que a intensidade e, consequentemente, a captura de cães em vias públicas parecem estar associadas aos baixos índices sociais locais (SHIMOZAKO et al, 2006) e a maior preocupação envolvendo a transmissão de zoonoses diz respeito aos cães parcialmente restritos já que estes têm contato com os animais efetivamente não domiciliados e, também, contato muitas vezes íntimo, com seus tutores (MATOS, 2002).

A principal estratégia utilizada, atualmente, no enfrentamento do problema da superpopulação de cães é a esterilização gratuita ou subsidiada. Diversos municípios já adotaram essa estratégia (DILLY et al, 2005; BORTOLOTI; D'AGOSTINO, 2007; MASCARENHAS et al, 2009; GOMES, 2010), mas estudo desenvolvido por Molento et al (2007) evidenciou a necessidade de se aumentar a expectativa de vida dos animais submetidos à castração, pois, caso contrário, haverá grande rotatividade e os castrados serão rapidamente substituídos por outros não castrados, já que a expectativa de vida desses animais é baixa. De fato, Almeida et al (2006) diagnosticaram que os cães não domiciliados do município de São Paulo (SP) possuíam baixa qualidade de vida e encontravam-se suscetíveis a diversas enfermidades, inclusive às zoonoses.

O objetivo do presente trabalho foi descrever as ações visando ao controle populacional de cães não domiciliados desenvolvidas em municípios do Estado do Rio de Janeiro, Brasil, no período 2012-2013.

\section{Material e métodos}

O Estado do Rio de Janeiro, situado na Região Sudeste, está dividido em 92 municípios e, no âmbito do Sistema Único de Saúde (SUS), é dividido em dez Regiões de Saúde, de acordo com o Plano Diretor de Regionalização estadual (RIO DE JANEIRO, 2001; COMISSÃO INTERGESTORES BIPARTITE, 2009).

Foi considerado como universo amostral para fins de definição da amostra de municípios a serem pesquisados, o conjunto dos 92 municípios que compõem o Estado do Rio de Janeiro. A partir desse universo foi utilizada a fórmula para estudo de proporções proposta por Thrusfield (2004), a um nível de significância de $95 \%$, frequência esperada de $50 \%$ e erro máximo permitido de $10 \%$, para a determinação da quantidade de municípios a serem pesquisados, resultando numa amostra de 47 municípios. A amostra de 47 municípios foi estratificada (BARBETTA, 2012) utilizando-se como variável a proporção de municípios em cada Região de Saúde. A seleção dos municípios seguiu sorteio aleatório simples (tabela 1).

Tabela 1: Quantitativo de municípios selecionados para aplicação dos questionários de pesquisa por Região de Saúde do Estado do Rio de Janeiro

\begin{tabular}{lcc}
\hline Região de Saúde & $\begin{array}{c}\text { Total de } \\
\text { Municípios }\end{array}$ & $\begin{array}{c}\text { Quantitativo de } \\
\text { Municípios Selecionados }\end{array}$ \\
\hline Baía da Ilha Grande & 03 & 02 \\
Baixada Litorânea & 09 & 05 \\
Capital & 01 & 01 \\
Centro-Sul & 11 & 06 \\
Médio-Paraíba & 12 & 06 \\
Metropolitana I & 11 & 06 \\
Metropolitana II & 07 & 04 \\
Noroeste & 14 & 06 \\
Norte & 08 & 04 \\
Serrana & 16 & 07 \\
\hline Total & 92 & 47 \\
\hline
\end{tabular}

Os municípios selecionados foram visitados, entre os anos de 2012 e 2013, e os responsáveis pelos serviços de controle de zoonoses responderam a um questionário estruturado com perguntas fechadas, previamente testado, após assinarem o Termo de Consentimento Livre e Esclarecido. O referido questionário tinha como eixos estruturantes o levantamento de informações básicas sobre os gestores dos serviços de zoonoses e sobre as ações de controle populacional de cães não domiciliados. 
Os dados obtidos dos questionários foram codificados, tabulados em planilhas do software $\operatorname{Excel}^{\circledR}$ e analisados com técnicas de estatística descritiva, utilizando-se o estudo das frequências e a aplicação do teste do qui-quadrado para verificação da significância estatística, com comparação entre as regiões.

A pesquisa foi aprovada pelo Comitê de Ética em Pesquisa da Faculdade de Medicina / Hospital Universitário Antônio Pedro / UFF, parecer CAAE 0318.0.258.000-11.

\section{Resultados}

Somente 46,8\% (n=22) dos municípios analisados realizavam alguma ação relacionada ao controle populacional de cães não domiciliados. As principais ações desenvolvidas eram: as educativas sobre guarda responsável e controle populacional de cães, em 29,8\% ( $n=14)$ dos municípios, a esterilização gratuita, em $21,3 \%(n=10)$ e os projetos de adoção, em $14,9 \%(n=7)$ dos municípios. Além disso, $8,5 \%(n=4)$ faziam recolhimento de cães não domiciliados e manutenção destes em canil, 4,2\% ( $n=2)$ subsidiavam esterilizações e 4,2\% $(n=2)$ realizavam outro tipo de ação de controle (figura 1). Nenhum dos municípios avaliados realizava recolhimento e posterior eutanásia como estratégia de controle populacional de cães não domiciliados.

Na tabela 2 podem ser observadas as ações desenvolvidas pelos municípios, divididas por região de saúde. As diferenças encontradas entre as regiões foram estatisticamente significantes $(p<0,05)$.
Em 53,2\% (n=25) dos municípios não era realizada nenhuma ação visando ao controle populacional de cães não domiciliados. Quando analisado por região de Saúde, percebe-se que a ausência de ações de controle populacional ocorria em 80\% ( $n=4)$ dos municípios da Baixada Litorânea, 75\% (n=3) da Metropolitana II, 71\% $(n=5)$ da Serrana, 66,7\% $(n=4)$ da Metropolitana I, 50\% da Noroeste $(n=3)$ e da Norte $(n=2)$ e $33,3 \%$ da Centro-Sul $(n=2)$ e da Médio Paraíba $(n=2)$.

Importante salientar que nenhum dos municípios avaliados:

- realizava ações educativas sobre guarda responsável nas regiões Centro-Sul e da Baixada Litorânea;

- realizava esterilização gratuita nas regiões da Baixada Litorânea, Metropolitana I, Metropolitana II e Noroeste.

- realizava recolhimento e manutenção em canil nas regiões da Capital, Baía da Ilha Grande, Baixada Litorânea, Centro-Sul, Metropolitana I, Metropolitana II e Norte.

- realizava projeto de adoção nas regiões da Baixada Litorânea, Centro-Sul, Metropolitana II, Norte e Noroeste.

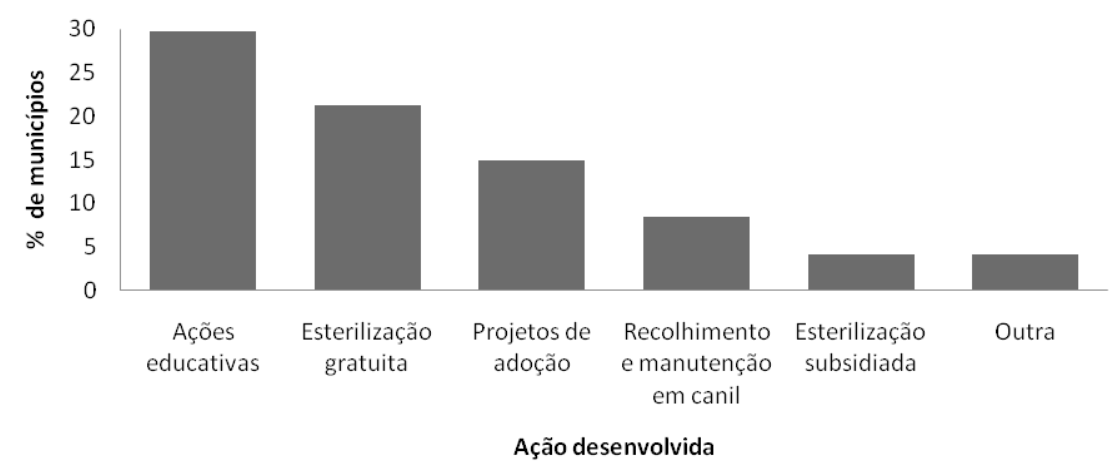

Figura 1: Proporção das ações de controle populacional de cães não domiciliados identificadas nos municípios, Estado do Rio de Janeiro, Brasil, 2013-2014.

Tabela 2: Quantitativo de municípios avaliados que desenvolviam ações de controle populacional de cães não domiciliados, por Região de Saúde, no Estado do Rio de Janeiro, Brasil, 2012-2013

\begin{tabular}{|c|c|c|c|c|c|c|c|c|c|c|c|c|}
\hline \multirow{3}{*}{ Região de Saúde } & \multicolumn{12}{|c|}{ Ação Desenvolvida } \\
\hline & \multicolumn{2}{|c|}{$\begin{array}{c}\text { Esterilização } \\
\text { gratuita }\end{array}$} & \multicolumn{2}{|c|}{$\begin{array}{c}\text { Esterilização } \\
\text { subsidiada }\end{array}$} & \multicolumn{2}{|c|}{$\begin{array}{l}\text { Recolhimento } \\
\text { e manutenção } \\
\text { em canil }\end{array}$} & \multicolumn{2}{|c|}{$\begin{array}{l}\text { Projeto de } \\
\text { adoção }\end{array}$} & \multicolumn{2}{|c|}{$\begin{array}{c}\text { Ação } \\
\text { educativa }\end{array}$} & \multicolumn{2}{|c|}{ Outra } \\
\hline & $\mathrm{n}$ & $\%$ & $\mathrm{n}$ & $\%$ & $\mathrm{n}$ & $\%$ & $\mathrm{n}$ & $\%$ & $\mathrm{n}$ & $\%$ & $\mathrm{n}$ & $\%$ \\
\hline Capital & 1 & 100 & 1 & 100 & 0 & 0 & 1 & 100 & 1 & 100 & 0 & 0 \\
\hline Baía Ilha Grande & 1 & 50 & 1 & 50 & 0 & 0 & 1 & 50 & 1 & 50 & 0 & 0 \\
\hline Baixada Litorânea & 0 & 0 & 0 & 0 & 0 & 0 & 0 & 0 & 0 & 0 & 1 & 20 \\
\hline Centro-Sul & 6 & 66,7 & 0 & 0 & 0 & 0 & 0 & 0 & 0 & 0 & 0 & 0 \\
\hline Médio Paraíba & 2 & 33,3 & 0 & 0 & 2 & 33,3 & 3 & 50 & 4 & 66,7 & 0 & 0 \\
\hline Metropolitana I & 0 & 0 & 0 & 0 & 0 & 0 & 1 & 16,7 & 2 & 33,3 & 0 & 0 \\
\hline Metropolitana II & 0 & 0 & 0 & 0 & 0 & 0 & 0 & 0 & 1 & 25 & 1 & 25 \\
\hline Noroeste & 0 & 0 & 0 & 0 & 1 & 16,7 & 0 & 0 & 2 & 33.3 & 0 & 0 \\
\hline Norte & 1 & 25 & 0 & 0 & 0 & 0 & 0 & 0 & 2 & 50 & 0 & 0 \\
\hline Serrana & 1 & 14,3 & 0 & 0 & 1 & 14,3 & 1 & 14,3 & 1 & 14,3 & 0 & 0 \\
\hline Total & 10 & 21,3 & 2 & 4,2 & 4 & 8,5 & 7 & 14,9 & 14 & 29,8 & 2 & 4,2 \\
\hline
\end{tabular}


Apenas dois municípios, sendo um a Capital e outro localizado na região da Baía da Ilha Grande realizavam esterilização subsidiada.

\section{Discussão}

As atividades educativas com a temática guarda responsável foram as mais realizadas pelos municípios, mas ainda assim com baixa adesão $(29,8 \% / n=14)$. A ação educativa, quando desenvolvida com planejamento, metodologia e frequência adequados pode ser considerada uma estratégia muito importante no enfrentamento do problema da superpopulação de cães não domiciliados, apesar de só ter efeito a longo prazo (INTERNATIONAL COMPANION ANIMAL MANAGEMENT, 2007). O fomento a estas atividades pode contribuir para a redução da quantidade de animais não domiciliados bem como a diminuição da incidência de zoonoses. Considerando que a ecologia das populações caninas está diretamente relacionada às ações humanas, deve-se investir na mudança do comportamento humano em relação aos cães (ORGANIZAÇÃO MUNDIAL DE SAÚDE ANIMAL, 2013). Diversos estudos destacam esta importância e sugerem a implantação ou intensificação das ações educativas (CARAMORI JÚNIOR, 2003; DOMINGOS et al, 2007; LIMBERTI et al, 2009; SILVA et al, 2009; GARCIA et al, 2012). Municípios como Resende já dispõem de legislação que obriga a municipalidade a desenvolver ações educativas em relação à Guarda Responsável (RESENDE, 2007). Além disso, deve-se considerar que as ações educativas de cunho coletivo não demandam grandes investimentos e as ONGs e voluntários podem ser envolvidos em sua realização (GOMES, 2010). Mas é de grande importância destacar que a ação educativa de maneira isolada não é capaz de solucionar o problema da superpopulação de cães não domiciliados, ou seja, ela é uma das estratégias necessárias ao enfrentamento multidisciplinar e multisetorial do problema em tela.

Proporcionalmente, a segunda ação mais desenvolvida pelos municípios foi a esterilização gratuita de cães $(21,3 \%$; $n=10)$. Considera-se a esterilização uma boa estratégia no conjunto de ações de controle populacional, principalmente pela sua segurança (CARVALHO et al, 2007) e pela possibilidade de ser realizada com baixo custo (MIGLIARI; DE VUONO, 2000; PEIXOTO et al, 2002). Os resultados encontrados são semelhantes aos de Gomes et al (2011) que constataram que 22,8 \% dos municípios avaliados no Estado de São Paulo realizavam esterilização. É importante que a esterilização seja gratuita pois o custo pode se tornar um fator impeditivo para as pessoas mais carentes (GARCIA, 2009). Mas a esterilização não pode ser uma estratégia isolada, dada a complexidade da ecologia das populações animais no ambiente antropizado. Estudo realizado por Molento et al (2007) sugere que a solução para o controle populacional passe, necessariamente pela educação para a guarda responsável, pois ao avaliar um projeto de esterilização no Paraná, os resultados mostraram alta taxa de mortalidade dos animais esterilizados e alto fluxo de moradores, fazendo com que em médio prazo a população de cães crescesse de tamanho, ainda que numa proporção pequena. Estudo de Amaku et al (2009) mostrou, baseado em simulação matemática, que a manutenção de um programa com altas taxas de esterilização ao longo de cerca de cinco anos pode levar à redução de $20 \%$ da densidade populacional canina, na hipótese de não haver importação de animais de outras áreas. O próprio Conselho Federal de Medicina Veterinária já dispõe de normativa que regulamenta os chamados Programas de Educação em Saúde, Guarda Responsável e Esterilização, os quais envolvem a realização de esterilização de cães e gatos, precedida ou associada a ações de educação (CONSELHO FEDERAL DE MEDICINA VETERINÁRIA, 2012).

A realização de projetos de adoção foi identificada em 14,9\% $(n=7)$ dos municípios. Esse resultado é menor que o encontrado por Gomes et al (2011) para o Estado de São Paulo, onde projetos de adoção eram realizados por $24,5 \%$ dos municípios. Ainda que não obrigatório por força de lei, é interessante que os animais disponibilizados para adoção estejam castrados, no intuito de contribuir para o controle populacional (ORGANIZAÇÃO MUNDIAL DE SAÚDE ANIMAL, 2013). Projetos de adoção, quando bem organizados, podem trazer bons resultados. Em estudo realizado no município de Carpina, PE, $56,3 \%$ de um total de 402 cães retirados das ruas por uma ONG de proteção animal foram adotados em eventos periodicamente realizados (VASCO NETO et al, 2009). Apesar disto, há exemplos de insucesso, como no caso de lbiúna, SP, em que somente cerca de $10 \%$ foram adotados num período de 8 meses (SOTO et al, 2006) e em Curitiba, PR, onde em cinco anos somente $12,4 \%$ dos cães apreendidos foram adotados (BIONDO et al, 2007).

Apenas $8,5 \%(n=4)$ dos municípios informaram realizar recolhimento e manutenção em canil de cães não domiciliados, resultado bem inferior ao encontrado por Gomes et al (GOMES et al, 2011) para o Estado de São Paulo, onde 35,3\% dos municípios faziam recolhimento desses cães, mas os autores não deixaram claro qual o destino dado aos animais recolhidos. O recolhimento e manutenção em canis em longo prazo torna-se extremamente custoso (INTERNATIONAL COMPANION ANIMAL MANAGEMENT, 2007) e de difícil operacionalização, não possibilitando uma qualidade de vida adequada aos animais e até dificultando o processo de adoção, pelo mal estado comumente apresentado pelos animais (REICHMANN et al, 2000). A instalação de abrigos, além de não solucionar o problema, pode intensificá-lo, pois proprietários insatisfeitos com seus animais podem abandoná-los na entrada dessas instituições por saberem que lá eles serão cuidados (BIONDO et al, 2007; INTERNATIONAL COMPANION ANIMAL MANAGEMENT, 2007).

O recolhimento e posterior eutanásia de cães sadios não eram realizados por nenhum município. Além da OMS não mais recomendar a eutanásia no controle da população canina, por considerá-la cara e ineficaz (ORGANIZAÇÃO MUNDIAL DA SAÚDE, 1999) no Estado do Rio de Janeiro o uso da eutanásia não é permitido para o controle de populações de animais domésticos (RIO DE JANEIRO, 2006).

Por fim, o fato de mais da metade $(53,2 \% / n=25)$ dos municípios avaliados não realizarem nenhuma ação visando ao controle populacional de cães não domiciliados é muito preocupante e pode ser justificado pelo descaso (SANTANA; MARQUES, 1988), pela heterogeneidade social, política, econômica e cultural que acarreta respostas diferenciadas de acordo com cada realidade (GOMES et al, 2011), pela falta de informação sobre o assunto (GARCIA, 2005) e pela falta de uma política nacional para o setor (GARCIA et al, 2012). 


\section{Conclusões}

Os resultados demonstraram que mais da metade dos municípios avaliados não desenvolvia qualquer ação visando ao controle populacional de cães não domiciliados, consequentemente conclui-se que o tema não era uma política pública prioritária, em especial nas Regiões de Saúde Serrana, Metropolitana I, Metropolitana II, Litorânea, Norte e Noroeste.

As principais ações desenvolvidas eram as atividades educativas sobre guarda responsável, a esterilização gratuita e os projetos de adoção, mesmo assim presentes em menos

\section{Referências}

ALMEIDA, M. F.; MOURIZ, E. S. M.; BESSA, A. F.; CHAPOLLA, E. G. B.; DAURIA, S. R. N.; YAI, L. E. O.; MARTORELLI, L. F. A.; VANNI, R. A. Inquérito sorológico para raiva, leishmaniose e leptospirose e infecção por enteroparasitas em cães errantes do Município de São Paulo (SP). Rev. bras. Vigilância Sanitária, v. 2, p. 146-149, 2006.

AMAKU, M. A.; DIAS, R. A.; FERREIRA, F. Dinâmica populacional canina: potenciais efeitos de campanhas de esterilização. Rev. panam. salud publica., v. 25, n. 4, p. 300-304, 2009.

BARBETTA, P. A. Estatística aplicada às Ciências Sociais. 8 ed. Florianópolis: UFSC, 2012318 p.

BIONDO, A. W.; CUNHA, G. R.; SILVA, M. A. G.; FUJI, K. Y.; UTIME, R. A.; MOLENTO, C. F. M. Carrocinha não resolve. Rev. Conselho Regional de Medicina Veterinária - PR, v. 5, n. 25, p. 20-21, 2007.

BORTOLOTI, R.; D'AGOSTINO, R. G. Ações pelo controle reprodutivo e posse responsável de animais domésticos interpretadas à luz do conceito de metacontingência. Rev. bras. aná. comport., v. 3, n. 1, p. 17-28, 2007.

CARAMORI JÚNIOR, J. G.; LUBAS, M. A. S.; KAWATAKE, M. S.; SALES, K. G.; GUEDES, J. C.; SCHMITT, A. C. Inquérito epidemiológico sobre características da população canina e felina de um bairro próximo à zona rural em Cuiabá-MT, visando o controle da raiva animal. Rev. soc. bras. med. trop., v. 38, n. 3, p. 419-420, 2003.

CARVALHO, M. P. P.; KOIVISTO, M. B.; PERRI, S. H. V.; SAMPAIO, T. S. M. C. Estudo retrospectivo da esterilização em cães e gatos no município de Araçatuba, SP. Rev. ciên. ext., v. 3, n. 2, p. 82, 2007.

COMISSÃO INTERGESTORES BIPARTITE. Deliberação $n^{\circ} 753 / 2009$. Constituição do Comitê Gestor Regional da Capital. 2009. Disponível em < http://www.cib.rj.gov.br/deliberacoescib/104-2009/novembro/593-deliberacao-cib-no-0753-de-13-denovembro-de-2009.html >. Acesso em: 05 de agosto de 2011.

CONSELHO FEDERAL DE MEDICINA VETERINÁRIA. Declaração Universal dos Direitos dos Animais. Disponível em http://www.cfmv.org.br/portal/direitos_animais.php. Acesso em: 02 de maio de 2012.

DILLY, B. L. G.; COSTA JÚNIOR, N. B.; FREITAS, R. B.; FRANCESCHINI, E. M. Tratamento dado ao problema de abandono de cães na UNICAMP. Rev. ciên. amb. on-line., v. 5, n. 1, 2005. Disponível em: http://sistemas.ib.unicamp.br/be310/ index.php/be310/article/viewFile/23/11. Acesso em: 13 de outubro de 2009. de $30 \%$ dos municípios. Já o recolhimento sistemático com posterior eutanásia não é mais uma prática frequente no Estado do Rio de Janeiro, não tendo sido relatado por nenhum município avaliado.

De modo geral as poucas ações visando ao controle populacional de cães não domiciliados desenvolvidas pelos municípios avaliados, em todas as Regiões de Saúde, eram fragmentadas e não seguiam um protocolo de atuação com ações integradas. Acredita-se que a falta de uma política ou diretriz nacional voltada para a questão do controle populacional dos cães não domiciliados contribua para o quadro encontrado.

DOMINGOS, I. H.; RIGO, L.; HONER, M. R. Perfil da populações canina e felina no município de Campo Grande, MS. Ensaios e c., v, 11, n. 1, p. 97-113, 2007.

FORATTINI, O. P. Ecologia, epidemiologia e sociedade. 2 ed. São Paulo: Artes Médicas, 2004. 710 p.

GARCIA, R. C. Controle de populações de cães e gatos em área urbana: uma experiência inovadora na Grande São Paulo. Saúde Coletiva, v. 2, n. 5, p. 24-28, 2005.

GARCIA, R. C. M. Estudo da dinâmica populacional canina e felina e avaliação de ações para o equilíbrio dessas populações em áreas da cidade de São Paulo, SP, Brasil. Tese (Doutorado) Programa de Pós-Graduação em Epidemiologia Experimental Aplicada às Zoonoses. São Paulo: USP, 2009. 265 p.

GARCIA, R. C.; MALDONADO, N.; FERREIRA, F. Consolidação de diretrizes internacionais de manejo de populações caninas em áreas urbanas e proposta de indicadores para seu gerenciamento. Rev. panam. salud publica., v. 32, n. 2, p. 140144, 2012.

GOMES, N. S. C. Uma perspectiva da construção dos direitos da fauna doméstica do município de Belo Horizonte - MG. Rev. bras. direito animal, v. 5, n. 7, p. 285-305, 2010.

GOMES, L. H.; MENEZES, R. F.; ARANDA, C. M. S.; VIEIRA, P. A. Serviços municipais de controle de zoonoses no Estado de São Paulo: diagnóstico situacional. Bol. epidemiol. pau., v. 8, n. 96, p. 11-31, 2011.

HAFEZ, E. S. E. Reprodução animal. 6 ed. Barueri, SP: Manole, 1995. $582 \mathrm{p}$.

\section{INTERNATIONAL COMPANION ANIMAL MANAGEMENT}

- ICAM. Guia de controle humanitário da população canina. 2007. Disponível em: http://www.icam-coalition.org/downloads/ Humane_Dog_Population_Management_Guidance_Portuguese. pdf. Acesso em: 23 de julho de 2013.

LIMBERTI, B. N. P.; MENEZES, J. S.; FERNANDES, S. S. P. Estudo da tríade: educação sanitária, posse responsável e bemestar animal em animais de companhia em comunidades de baixa renda. Anuário de produção de iniciação científica discente, v. XII, n. 13, p. 99-108, 2009.

MASCARENHAS, N. M. F.; HILST, C. L. S.; SOUZA, M. S. B.; MARTINS, M. I. M.; PIROLO, J.; NABUT, L. B.; MACHADO, M. A.; NAVARRO, I. T.; FRÜHVALD, E.; ZANONI, F. P.; TOKIYOSHI, A. F.; LACERDA, L. C. C. Posse responsável e controle populacional de cães e gatos e sua contribuição para a redução da eutanásia na população errante de Londrina e região. $35^{\circ}$ Congresso Brasileiro de Medicina Veterinária 2008. In: Anais... Disponível em: www.sovergs.com.br/conbravet2008/anais/cd/resumos/ R0706-1.pdf. Acesso em: 13 de novembro de 2009. 
MATOS, M. R.; ALVES, M. C. G. P.; REICHMANN, M. L. A. B.; DOMINGUÉZ, M. H. S. Técnica Pasteur São Paulo para dimensionamento de população canina. Cad. saúde pública, v. 18, n. 5, p. 1423-1428, 2002.

MIGLIARI, R.; DE VUONO, R. S. Ovário salpingo-histerectomia em cadelas e gatas - proposta de novos procedimentos. Rev. educ. cont. CRMV-SP, v. 3, n. 3, p. 28-32, 2000.

MOLENTO, C. F. M.; LAGO, E.; BOND, G. B. Controle populacional de cães e gatos em dez vilas rurais do Paraná: resultados de médio prazo. Arch. vet. sci., v. 12, n. 3, p. 43-50, 2007.

ORGANIZAÇÃO MUNDIAL DA SAÚDE. O controle da raiva: oitavo relatório do comitê de especialistas da OMS em raiva. Tradução Fernando Melgaço de Assumpção Costa. 1 ed. Goiânia: Ed. UFG, 1999. 152 p.

ORGANIZAÇÃO MUNDIAL DE SAÚDE ANIMAL - OIE. Control de las poblaciones de perros vagabundos. In: OIE. Código Sanitario para los Animales Terrestres - 2013. Disponivel em: http://www. oie.int/es/normas-internacionales/codigo-terrestre/acceso-enlinea/. Disponível em: 22 de outubro de 2013.

PEIXOTO, E. C. T. M.; VICENTE, W. R. R.; SANTOS, M. I. A.; NETTO, T. R. HAYASHI, F. E. Controle populacional de pequenos animais: esterilização cirúrgica a preço mínimo. ARS vet., v. 18, n. 3, p. 258-266, 2002.

REICHMANN, M. L. A. B.; FIGUEIREDO, A. C. C.; PINTO, H. B. F.; NUNES, V. F. P. Controle de populações de animais de estimação. São Paulo: Instituo Pasteur, 2000. (Manuais, 6) 44p.

RESENDE-RJ. Lei $n^{\circ} 4383$ de 20 de setembro de 2007. Institui o Código Municipal de Proteção aos Animais, no âmbito do Município de Resende RJ, e dá outras providências. 2007. Disponível em: http://defensoresdosanimais.wordpress.com/ juridico-2/legislacao/legislacao-municipal/nova-2/. Acesso em: 27 de janeiro de 2014 .

RIO DE JANEIRO. Secretaria de Estado de Saúde. Plano Diretor de Regionalização: Secretaria de Estado de Saúde, 2001. 128 p.

RIO DE JANEIRO. Lei $n^{\circ} 4.808$ de 04 de julho de 2006. Dispõe sobre a criação, a propriedade, a posse, a guarda, o uso, o transporte e a presença temporária ou permanente de cães e gatos no âmbito do estado do Rio de Janeiro. Disponível em: http://alerjln1.alerj.rj.gov.br/contLei.nsf/c8aa0900025feef6032564 ec0060dfff/6628191723549496832571a8005e8896?opendocume nt. Acesso em: 24 de julho de 2010.
SANTANA, L. R.; MARQUES, M. R. Maus tratos e crueldade contra animais nos Centros de Controle de Zoonoses: aspectos jurídicos e legitimidade ativa do Ministério Público para propor Ação Civil Pública. 1998. Disponível em: http://www. forumnacional.com.br/maus_tratos_CCz_de_Salvador.pdf. Acesso em 14 de outubro de 2009.

SANTANA, L. R.; PITA, R. T. O.; ORLANDI, V. T.; GUSMÃO, P. A. M. Controle pelo Ministério Público e pelo Poder Judiciário das políticas públicas assecuratórias dos princípios e direitos constitucionais aplicáveis à dignidade e bem estar dos animais. 2008. Disponível em www.forumnacional.com.br/controle_pelo_ poder judiciario.pdf. Acesso em: 14 de outubro de 2009.

SHIMOZAKO, H. J.; AMAKU, M.; FERREIRA, F.; DIAS, R. A.; NETTO, H. M.; PARANHOS, N. T.; DIAS, RB. Uso de sistemas de informação geográfica como ferramentas de auxílio na análise de casos de apreensão de animais no município de São Paulo. Rev. ciênc. ext., v. 2, n. 2, p. 1-16, 2006.

SILVA, F. A. N.; CARVALHO, R. L.; KLEIN, R. P.; QUESSADA, A. $M$. Posse responsável de cães no bairro Buenos Aires na cidade de Teresina (PI). ARS vet., v. 25, n. 1, p. 14-17, 2009.

SOTO, F. R. M.; SOUZA, A. J.; RISSETO, M. R.; LIMA, B. F. M. $\mathrm{S}$. Adoção de cães no município de Ibiúna - SP - Brasil: análise crítica. Rev. ciên. ext., v. 3, n. 1, p. 27, 2006.

SOUZA, M. F. A. Controle de populações caninas: considerações técnicas e éticas. Rev. Bras. direito animal, v. 8, n. 6, p. 115-133, 2011.

THRUSFIELD, M. Epidemiologia veterinária. 2. ed. São Paulo: Roca, 2004. 556 p.

VASCO NETO, H. L. S.; SILVA, D. D.; SILVA, R. R. P.; ROSA, M. G. S. Ações sociosanitárias para incentivo a posse responsável de animais no município de Carpina - PE. IX Jornada de Ensino, Pesquisa e Extensão da UFRPE. 2009. In: Anais.... Disponível em: http://www.eventosufrpe.com.br/jepex2009/cd/resumos/ R0674-1.pdf. Acesso em: 02 de janeiro de 2014.

VIEIRA, A. M.L.; ALMEIDA, A. B.; MAGNABOSCO, C.;FERREIRA, J. C. P.; LUNA, S. L. P.; CARVALHO, J. L. B.; GOMES, L. H.; PARANHOS, N. T.; REICHMANN, M. L.; GARCIA, R. C.; NUNES, V. F. P.; CABRAL, V. B. Programa de controle de cães e gatos do Estado de São Paulo. Bol. epidemiol. pau., v. 3, n. 25, 2006. Disponível em: http://www.cve.saude.sp.gov.br/ agencia/bepa25_rg7caes.htm . Acesso em: 22 de fevereiro de 2012. 